\title{
Nontuberculous mycobacterial infection in rheumatoid arthritis patients: a single-center experience in South Korea
}

Doo-Ho Lim¹, Yong-Gil Kim¹, Tae Sun Shim², Kyung-Wook Jo², Byeongzu Ghang', Soo Min Ahn", Seokchan Hong ${ }^{1}$, Chang-Keun Lee ${ }^{1}$, and Bin Yoo ${ }^{1}$

Divisions of ${ }^{1}$ Rheumatology and ${ }^{2}$ Pulmonary and Critical Care Medicine, Department of Internal Medicine, Asan Medical Center, University of Ulsan College of Medicine, Seoul, Korea

Korean J Intern Med 2017;32:1090-1097

https://doi.org/10.3904/kjim.2015.357

In the article cited above, there was a spelling error in the main text. Sixth line of the third paragraph in RESULTS part, page 1092, "abatacept" was misspelled. The correct word is "adalimumab."

We apologize for any inconvenience that this may have caused. 